\title{
Variational analysis of driven-dissipative bosonic fields
}

\author{
Tim Pistorius and Hendrik Weimer"* \\ Institut für Theoretische Physik, Leibniz Universität Hannover, Appelstraße 2, 30167 Hannover, Germany
}

\begin{abstract}
We present a method to perform a variational analysis of the quantum master equation for drivendisspative bosonic fields with arbitrary large occupation numbers. Our approach combines the $\mathrm{P}$ representation of the density matrix and the variational principle for open quantum system. We benchmark the method by comparing it to wave-function Monte-Carlo simulations and the solution of the Maxwell-Bloch equation for the Jaynes-Cummings model. Furthermore, we study a model describing Rydberg polaritons in a cavity field and introduce an additional set of variational paramaters to describe correlations between different modes.
\end{abstract}

\section{INTRODUCTION}

The theoretical analysis of driven-dissipative quantum many-body systems is a very challenging task, as many methods developed for closed quantum systems cannot be applied. While important insights have been obtained using the Wave-Function Monte-Carlo (WFMC) method [1, 2] or tensor network approaches [3 6], the numerical study of bosonic fields with large occupation numbers remains an outstanding problem [7]. Here, we show that the variational principle for open quantum systems [8] provides for a natural way to represent arbitrarily large occupation numbers in terms of a small set of variational parameters, thus providing a highly efficient description of the system.

Driven-dissipative systems of bosons arise in many different settings, ranging from semiconductor polaritonic systems 9], to cavity quantum electrodynamics arrays 10, to Rydberg polaritons in atomic quantum gases [11, 12]. Especially for the latter, the development of efficient numerical descriptions is of great interest due to the applications of Rydberg polaritons in the context of strongly correlated photon states [13] and photonic quantum computing $14-16$.

In this article, we present a variational treatment of the steady state of driven-dissipative bosons. While the Lindblad formalism allows to calculate the steady state based on the solution of a quantum master equation [17, a brute-force solution becomes prohibitive for large Hilbert space dimensions. Here, we apply the variational principle for open quantum systems, which already proved to be a reliable method in the context of spin- $\frac{1}{2}$ particles [8, 18, 19. However, a direct implementation of the variational principle for bosonic fields results in a large amount of variational parameters to describe large Hilbert space, which drastically reduces the efficiency of the method and still relies on a cut-off of the Hilbert space. Therefore, we turn to a different implementation of the variational principle based on the GlauberSudarshan $\mathrm{P}$ representation.

Phase-space representations of the density matrix have received considarble interest in recent years to classify the

\footnotetext{
* hweimer@itp.uni-hannover.de
}

nonclassical properties of quantum states [20 26]. For this, the $\mathrm{P}$ representation by Glauber and Sudarshan $[27$. 28. is particularly useful, as negative values directly point to nonclassical behavior.

In this following, we want to use this representation of the density matrix to expand the variational method for open quantum systems to bosonic fields. We also show how the formalism of the variational principle translates to the Heisenberg equations of motions and how this can be used to extend the method to highly singular $\mathrm{P}$ distribution where an explicit representation is not feasible. We benchmark our approach by comparing to WFMC simulations 29, 30 mean-field calculations for the Jaynes-Cummings model [31-34. We also study a highly correlated model describing Rydberg polaritons in a cavity field. There, we introduce additional variational parameters to also implement correlation between different modes. Finally, we give an outlook how to extend our approach to incorporate other nonclassical states.

\section{VARIATIONAL METHOD}

Our method is based on the idea to use the $\mathrm{P}$ representation of the density matrix operator to express an variational state for a bosonic field for a minimazation process. First, we want to give a brief introduction to both concepts and then we show how to combine them.

In the context of open quantum systems, states are commonly described in terms of their density operator $\rho$. The Markovian dynamics of such open quantum systems can then be described by the Lindblad master equation $\frac{d}{d t} \rho=\mathcal{L} \rho$, with the Liouvillian $\mathcal{L}$ being the generator of the dynamics for the density matrix $\rho$ 35], i.e.

$\frac{d}{d t} \rho(t)=\mathcal{L}(\rho)=-i[H(t), \rho(t)]+\sum_{i}\left(c_{i} \rho c_{i}^{\dagger}-\frac{1}{2}\left\{c_{i}^{\dagger} c_{i}, \rho\right\}\right)$.

At the same time, the equation of motions can also be expressed in the Heisenberg picture, i.e., for any given obserable $A$ as

$$
\left.\frac{d}{d t} \hat{A}(t)=i[H, \hat{A}]+\sum_{i}\left(c_{i} \hat{A} c_{i}^{\dagger}-\frac{1}{2} c_{i}^{\dagger} c_{i}, \hat{A}\right\}\right) .
$$

In both equations, the jump operators $c_{i}$ correspond to 
incoherent processes, e.g. dephasing or dissipation, between the system and the bath.

Solving Eq. (1) is often very challenging [7, i.e., typically some approximations have to be made. Within the variational principle for open quantum systems [8], the density matrix is approximated by the usage of a variational ansatz. In case we want to solve for the steady state of the system $(\dot{\rho}=0)$ we need to minimize

$$
\left\|\dot{\rho}_{\text {var }}\right\|=\left\|\mathcal{L}\left(\rho_{\text {var }}\right)\right\| \rightarrow \min
$$

with $\|\dot{\rho}\|=\operatorname{Tr}\{|\dot{\rho}|\}$ being the trace norm. Here, we want to define a similar approach in the Heisenberg picture, where the steady state is defined as $\frac{d}{d t}\langle\hat{A}\rangle(t)=0$ for all operators $\hat{A}$. Therefore, we determine the steady state by minimizing a suitable norm for a small subset of operators given by

$$
\sum_{n}\left|\frac{d}{d t}\left\langle\hat{A_{n}}\right\rangle^{\mathrm{var}}(t)\right|=\sum_{n}\left|\mathcal{L}\left(\left\langle\hat{A_{n}}\right\rangle^{\mathrm{var}}\right)\right| \rightarrow \min .
$$

with $\left\langle\hat{A_{n}}\right\rangle^{\text {var }}$ being the $n$th variational expectation value $\operatorname{Tr}\left\{\rho_{\text {var }} \hat{A_{n}}\right\}=\left\langle\hat{A_{n}}\right\rangle^{\mathrm{var}}$. In the next paragraph, we will discuss how the $\mathrm{P}$ representation can be used to construct $\rho_{\text {var }}$ for bosonic fields.

The most straight forward implementation of a variational density matrix is to use each entry of the matrix as a variational parameter. This is, however, not feasible in most cases. In the case of an infinite Hilbert space the number of variational paramters also goes to infinity. A solution for this problem can be found in the $\mathrm{P}$ representation of the density matrix 27.

$$
\rho=\int d^{2} \alpha P(\alpha)|\alpha\rangle\langle\alpha| .
$$

The non-orthogonality of coherent states form an overcomplete basis set of states which we can use to represent the density matrix if we combine them with an appropriate choice of quasiprobability distribution $P(\alpha)$. Such a distribution can theoretically be found for any kind of density matrix [36] if we allow the class of generalized function in our distribution. This excludes any interpretation as an analogue to classical distribution functions because $P(\alpha)$ can become negative or more singular than a Dirac delta function $\delta(x)$.

A useful property of this specific representation is the way how expectation values of annihaltion (creation) operators $a\left(a^{\dagger}\right)$ are calculated through $c$-number integrals

$$
\left\langle: a^{\dagger p} a^{q}:\right\rangle=\operatorname{Tr}\left\{\rho a^{\dagger p} a^{q}\right\}=\int d^{2} \alpha P(\alpha) \alpha^{* p} \alpha^{q},
$$

where $\left\langle: a^{\dagger p} a^{q}:\right\rangle$ indicates normal ordering of the operators. We will drop the indicator for normal ordering and always assume that the expectation values are in that order for the rest of the paper.

We also want to consider the combination of different quantum states to expand the variational manifold. The construction of the corresponding $\mathrm{P}$ distribution is done by a convolution

$$
P(\alpha)=\left(P_{i} * P_{j}\right)(\alpha)=\int d \alpha d \alpha^{\prime} P_{i}\left(\alpha^{\prime}\right) P_{j}\left(\alpha-\alpha^{\prime}\right)
$$

of the original distributions $P_{i}$ and $P_{j}$ [37. If we insert (7) into (6) we obtain

$$
\left\langle a^{\dagger p} a^{q}\right\rangle=\sum_{n}^{p} \sum_{m}^{q} \xi_{p, q}\left\langle\left(a^{\dagger}\right)^{n} a^{m}\right\rangle_{P_{i}}\left\langle\left(a^{\dagger}\right)^{p-n} a^{q-m}\right\rangle_{P_{j}}
$$

to compute expectation values of a convoluted $\mathrm{P}$ distribution with $\xi_{n, m}$ as the number of possible combinations of the given expectation values from $\left\langle a^{\dagger n} a^{m}\right\rangle$. For example, if we assume that one of the distribution are for the thermal state we regain the same result as in $[38$. We see that the calculation depends on all expectation values up to the orders $p, q$ of $a, a^{\dagger}$ of the orignial expectation value but are calculated for the single $\mathrm{P}$ distributions $P_{i}$ and $P_{j}$. This process can then be repeated multiple times to combine multiple distributions. Table \in App. A shows examples of the convolution of two different states.

These ingredients are all that is required to formulate the variational principle in terms of the $\mathrm{P}$ distribution. The equations of motion created by Eq. (2) for the expectation value $\hat{A}$ depend only on expectation values like

$$
\frac{d}{d t}\langle\hat{A}\rangle(t)=F\left(\left\{\left\langle a^{\dagger p} a^{q}\right\rangle\right\}_{p, q}\right) .
$$

This means that we can write Eq. (4) as

$$
D=\sum_{i} F_{i}\left(\left\{\left\langle a^{\dagger p} a^{q}\right\rangle_{p, q}\right\}\right) \rightarrow \min
$$

with $F_{i}$ describing the right hand side of Eq. (2), which depends on the set of expectation values $\left\{\left\langle a^{\dagger p} a^{q}\right\rangle\right\}_{p, q}$. To see how the $\mathrm{P}$ representation can be used to describe these expectation values in terms of variational paramaters, it is instructive to have a look at some well known cases for $P(\alpha)$. First, we consider two classical states, a coherent and a thermal state, represented by

$$
\begin{aligned}
& P_{\text {coherent }}(\alpha)=\delta\left(\alpha-\alpha_{0}\right) \\
& P_{\text {thermal }}(\alpha)=\frac{1}{\pi n_{0}} \exp \left(-\frac{|\alpha|^{2}}{n_{0}}\right) .
\end{aligned}
$$

In addition, we can find a expression for a highly nonclassical state in form of the Fock states that look like

$$
P_{\text {fock }}=\frac{1}{l !} e^{|\alpha|^{2}} \frac{\partial^{2 l}}{\partial \alpha^{l} \partial \alpha^{* l}} \delta^{(2)}(\alpha)
$$

The distribution includes derivatives of the delta distribution which are defined as $\int d x \delta^{(n)}(x) \psi(x)=$ $(-1)^{n} \psi^{(n)}(0)$. We can immediately see that each distribution has one defining parameter, i.e., $\alpha_{0} \in \mathbb{C}, n_{0} \in \mathbb{R}$, 
or $l \in \mathbb{N}$. The convolution of two $\mathrm{P}$ distributions results in a new $\mathrm{P}$-distribution that depends on the set $\{\beta\}=\alpha_{0}, n_{0}, l$. This allows us to formulate Eq. 10 as

$$
D=\sum_{i} F_{i}(\{\beta\}) \rightarrow \min
$$

Upon inspection of Eq. (8) we can also see that we do not need to know the complete form of the $\mathrm{P}$ distribution that corresponds to a specific state. Instead, it is enough to know how all expectation values depend on the variational parameters $\beta$. This is for example useful if it is difficult to find a complete expression of the $\mathrm{P}$ distribution like in the case of the squeezed coherent state. The state can be obtained by convolution of the coherent state and the squeezed vacuum state where the distribution is known [39] or by directly evaluating the expectation values for this particular state. In this case we know that the squeezing operator $S^{(\dagger)}(r, \Phi)$ with squeezing parameter $r$ and angle $\Phi$ changes the annihaltion operator $\hat{a}$ like

$$
S^{\dagger} \hat{a} S=\hat{a} \cosh (r)-e^{i \Phi} \hat{a}^{\dagger} \sinh (r)
$$

which allows us to directly calculate how the expectation values in Eq. (10) depend on the parameters $r$ and $\Phi$.

\section{JAYNES-CUMMINGS MODEL}

In order to benchmark our variational approach, let us turn to a driven-dissipative variant of the JaynesCummings model, where we compare the variational method to WFMC simulations using the QuTiP package [29, 30]. The Jaynes-Cummings model describes a atom interacting with a light field that is trapped inside a cavity. The Hamiltonian is of the form

$$
H=\Delta_{c} a^{\dagger} a+\Delta_{a} \sigma^{+} \sigma^{-}+g\left(a \sigma^{+}+a^{\dagger} \sigma^{-}\right)+p\left(a^{\dagger}+a\right) .
$$

The first two terms describe the detunings $\Delta_{c}, \Delta_{a}$ for the cavity respectivly the atoms from the driving frequency. The atom and the cavity are coupled with a strength $g$ and we pump the cavity with an driving amplitude $p$. Additionally, we include cavity losses and spontaneous emission of the atoms into other modes than the cavity via the jump operators $c_{c}=\sqrt{\gamma} a$ and $c_{a}=\sqrt{\kappa} \sigma^{-}$with decay rate $\gamma$ for the cavity mode and $\kappa$ for the atom, respectively.

We use a product ansatz for the atom and the cavity

$$
\rho=\rho_{\text {cavity }} \otimes \rho_{\text {atom }}
$$

in the variational approach and use the variational parameter $\alpha_{i}$ in $\sum_{i=0, x, y, z} \alpha_{i} \sigma_{i}$ to describe the atomic part, while we use the $\mathrm{P}$ representation to account for the cavity mode. As our variational parameter set we use a convolution of coherent, thermal, fock and squeezed states.

To show an immediate advantage of the variational approach, we also want to analyse the Maxwell-Bloch equations of the Jaynes-Cummings model [32, 34. This set of

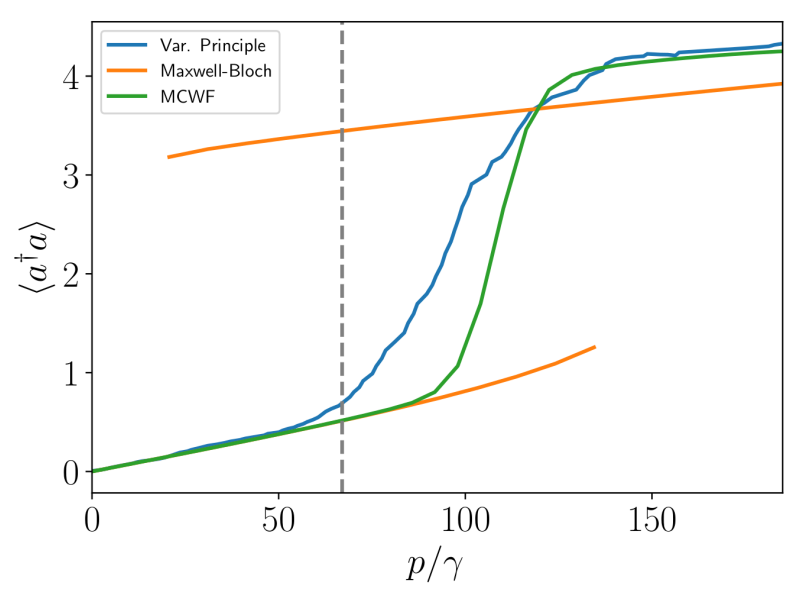

FIG. 1. Results of the Maxwell-Bloch equations, the variational approach and the Monte-Carlo wavefunction method (MCWF) for the Jaynes-Cummings model with $g / \gamma=3347$, $\kappa / \gamma=6, \Delta_{c} / \gamma=340, \Delta_{a} / \gamma=23.5 \times 10^{3}$. The Maxwell-Bloch equations (orange) show a region of bistability which can be solved by a variational treatment of the equations which yields a prediction for the transition between the two solution at the grey line. For higher orders of the variational approach (blue) shifts the transition towards the MCWF solution.

equation describe the time evolution of the lowest order of expectation values. The atom and cavity decouples in a similar fashion like in Eq. (17), but it also decouples the equation from higher order terms of the cavity field through the neglection of any correlation term of the second or higher order [40]. The Maxwell-Bloch equations for the Jaynes-Cummings model are given by

$$
\begin{aligned}
\frac{d}{d t}\langle a\rangle & =-\left(\kappa+i \delta_{c}\right)\langle a\rangle-i g\left\langle\sigma^{-}\right\rangle-i p \\
\frac{d}{d t}\left\langle\sigma^{-}\right\rangle & =-\left(\frac{\gamma}{2}-i \Delta_{a}\right)\left\langle\sigma^{-}\right\rangle+i g\langle a\rangle\left\langle\sigma^{z}\right\rangle \\
\frac{d}{d t}\left\langle\sigma^{z}\right\rangle & =-\gamma\left(\left\langle\sigma^{z}\right\rangle+1\right)+2 i g\left(\left\langle a^{\dagger}\right\rangle\left\langle\sigma^{-}\right\rangle-\langle a\rangle\left\langle\sigma^{+}\right\rangle\right)
\end{aligned}
$$

Fig. 11 shows a comparison between the solution of the Maxwell-Bloch equations, the Monte-Carlo wave function solution and the variational expectation value approach for the cavity field $\left\langle a^{\dagger} a\right\rangle$. The mean-field solution (orange) shows a large area of bistability between two solutions. A comparison of the norms of the two solutions inserted in a set of first-order equations of motion resolves the bistability and indicates a jump between the solutions at the grey line. The third line (blue) shows the solution of expanding the equations of motion up to second order in the variational approach, resulting in a clear improvement. Fig. 2 shows a reconstructed P distribution from the variational expectation values through 


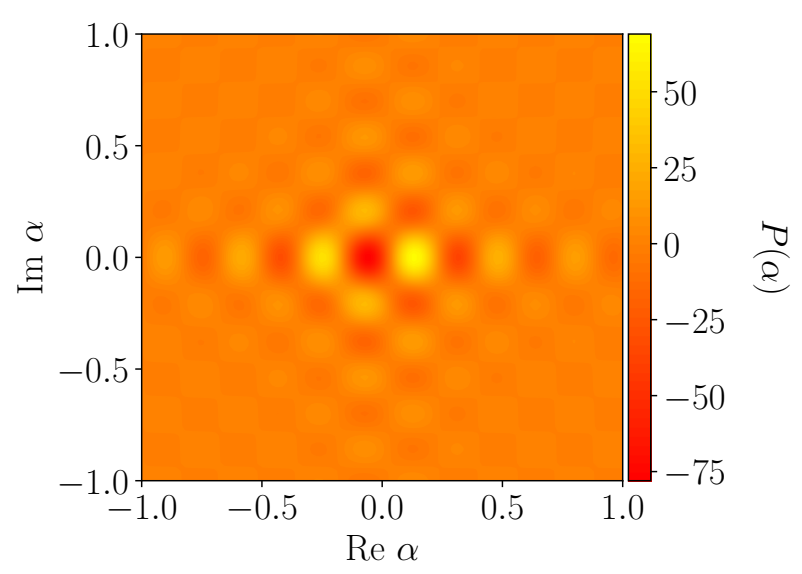

FIG. 2. P-distribution of the Jaynes-Cummings model for $g / \gamma=3347, \kappa / \gamma=6, \Delta_{c} / \gamma=340, \Delta_{a} / \gamma=23.5 \times 10^{3}$ and $p / \gamma=50$. The distribution shows regions of strong negativity which indicates a non-classical behavior of the model for the given parameters. The distribution is obtained via the variational principle.

the usage of the characteristic function

$$
\chi(z)=\sum_{k, l=0}^{\infty} \frac{z^{k}}{k !} \frac{\left(-z^{*}\right)^{l}}{l !}\left\langle a^{\dagger k} a^{l}\right\rangle
$$

and

$$
P\left(\alpha, \alpha^{*}\right)=\frac{1}{\pi^{2}} \int_{-\infty}^{\infty} d^{2} z \chi(z) e^{-i z^{*} a^{\dagger}} e^{-i z a} .
$$

The non-classicality of the steady state is clearly shown by the negative values of $P(\alpha)$. The remaining difference with the WFMC simulations can be attributed to the neglection of correlations between the atom and the cavity mode due to our product ansatz in Eq. (17).

\section{RYDBERG CAVITY POLARITONS}

Let us now turn to a model where correlations beyond a single mode are particularly important. For this, we investigate an effective three-boson model to describe strongly interacting Rydberg atoms inside a cavity [41, 42, which describes nonlinear effects that arises from the interaction of the Rydberg atoms. Before turning to the variational analysis, we briefly want to recapture the key pieces of the model.

Consider a cavity filled with $N$ three-level atoms with energy level $g, e, r$ as the ground state $|g\rangle$, an intermediate level $|e\rangle$ and an highly excited state which we denote as the Rydberg level $|r\rangle$. The key idea is to restrict the dynamics to three bosonic modes that describe the cavity mode and the symmetric subspaces of the atomic excitations. This restriction of the atoms to their symmetric

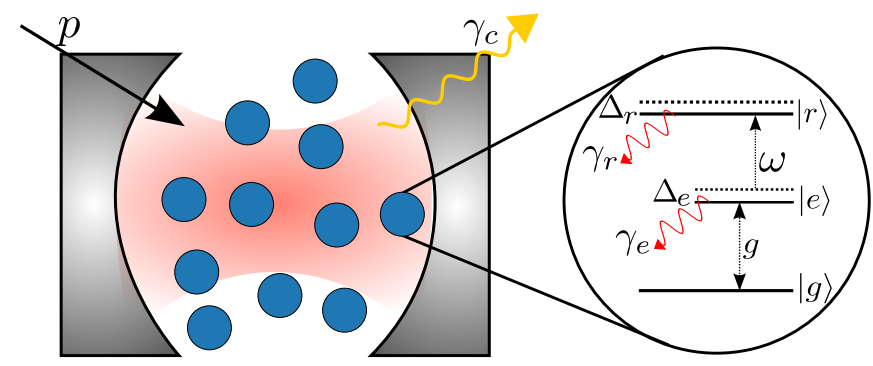

FIG. 3. Scheme of multiple Rydberg atoms trapped inside a cavity. The atoms are described by a three-level ladder scheme with $g$ as the coupling constant between the cavity light field and the transition between the ground state $|g\rangle$ and the intermediate state $|e\rangle$. A control laser then couples the intermediate to the Rydberg state $|r\rangle$ with a strength of $\omega$. The one- and two-photon detunings of the atoms are given by $\Delta_{e}$ and $\Delta_{r}$. The rates $\gamma_{c}, \gamma_{e}$ and $\gamma_{r}$ describe decay processes of the cavity, the intermediate atomic state and the Rydberg state.

subspace is valid as long as the total number of atomic excitations is small compared to $N$ [42].

We then can describe the system in terms of collective operators describing the symmetric subspace with $a$ being the annihilation operator for the cavity mode and $b=\frac{1}{\sqrt{N}} \sum_{n=1}^{N} \sigma_{g e}$ and $c=\frac{1}{\sqrt{N}} \sum_{n=1}^{N} \sigma_{g r}$ as the collective operators for the atomic transition modes $\sigma_{g e}$ and $\sigma_{g r}$. With that the Hamiltonian reads as

$$
\begin{aligned}
H & =-\Delta_{c} a^{\dagger} a+p\left(a+a^{\dagger}\right)-\Delta_{e} b^{\dagger} b-\Delta_{r} c^{\dagger} c \\
& +g \sqrt{N}\left(a b^{\dagger}+a^{\dagger} b\right)+\frac{\omega}{2}\left(b c^{\dagger}+b^{\dagger} c\right)+\frac{\kappa_{r}}{2} c^{\dagger} c^{\dagger} c c
\end{aligned}
$$

and the the jump operators are given by $c_{e}=\sqrt{\gamma_{e}} b, c_{r}=$ $\sqrt{\gamma_{r}} c$ for the intermediate and Rydberg state and also for the cavity. The nonlinear terms in the Hamiltonian arise from the van-der-Waals-force between atoms in the Rydberg state. The interaction also couples the symmetric subspace to the antisymmetric subspace which leads to an additional nonlinear dissipation term $c_{n l}=\sqrt{\kappa_{i}} c c$.

We now study the model by working in the eigenbasis of the non-interacting Hamiltonian at $\kappa_{r}=0$. The diagonalisation of Eq. 23] results in $H=\sum_{q \in+, 0,-} c_{q} \Psi_{q}^{\dagger} \Psi_{q}$. The new states $\Psi_{q}$ form polariton states. There are defined as a quasi particle consisting of both light and matter. For a three level atomic system we get two different types of polaritons. The ones with $q \in \pm$ are bright state polaritons, while $q=0$ is the dark state polariton. The dark state polariton shows very different behavior as it is decoupled from the intermediate atomic level, which leads to long lifetimes in the cavity.

The previously neglected interaction between the polariton leads to a strongly correlated many-body system which provides a difficult task for numerical calculation especially for large atom numbers [43].

To also be able to capture correlations between the modes we need additional variational parameters. If we 

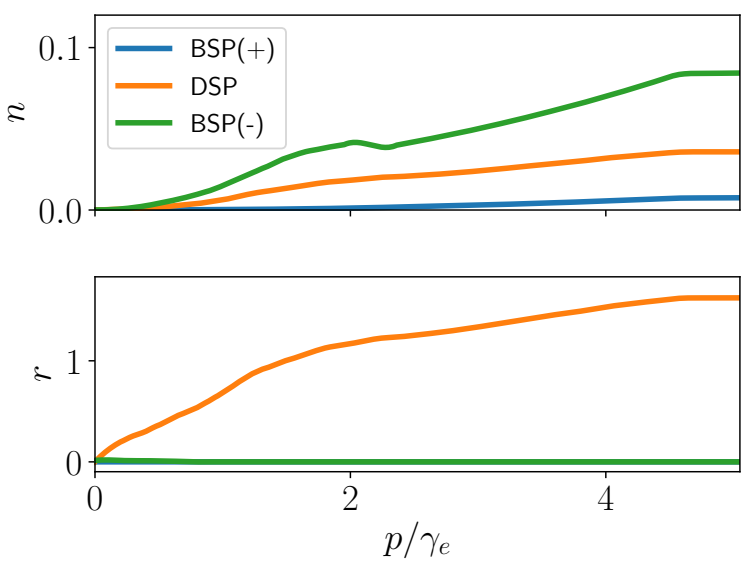

FIG. 4. Intensity(Top) and squeezing parameter $r$ (buttom) of the effective three boson model in the polariton picture for parameters: $\gamma_{r}=0.1 \gamma_{e}, \gamma_{c}=0.3 \gamma_{e}, \Delta_{c}=0, \Delta_{e}=-10 \gamma_{e}$, $\Delta_{r}=0, \kappa_{r}=-1.2 \gamma_{e}, \kappa_{i}=0.42 \gamma_{e}, g=4.2 \gamma_{e}, N=10^{4}$. The intensity of all polariton modes increase with the pumping strength and reaches a saturation at around $p \approx 5 \gamma_{e}$. However, only the dark state polariton mode displays squeezing. For the bright state polaritons, the squeezing parameter essentially vanishes.

look at the lowest order expectation values between different modes we get

$$
\langle a b\rangle=\langle a\rangle\langle b\rangle+\delta(a b)
$$

with $\delta(a b)$ as the correlation function between mode $a$ and $b$. These kind of factorizations for expectation values can be done for all orders and provides us with the needed variational parameter in form of the correlation functions $\delta\left(a^{n} b^{m}\right)$ 44 46.

Fig. 4 shows the occupation number of the different modes and their squeezing strength as the parameter $r$. The photons are getting absorbed by the different photon modes. The bright state polaritons show for the offresonant parameters we have chosen here an uneven population. All modes reach a saturation around $p \approx 5 \gamma_{e}$. If we look at squeezing parameter we can see that this is the only mode that experiences strong nonlinear effects while the squeezing is mostly suppressed for the other two.

Our results also demonstrate the importance including correlations between the modes. Without them, we find that the occupation numbers can become very large (e.g., up to $n=200)$, which correspond to states with very large (and hence unphysical) van der Waals interaction energies.

\section{POSSIBLE EXTENSIONS}

In all of our previous calculations, we worked with only a handful of different convoluted states to successfully construct our variational manifold. However, we would like to point out that it is possible to extend our approach toeven broader classes of quantum states. As already mentioned, it is not necessary to know the full $\mathrm{P}$ distribution function as it is sufficient to be able to calculate expectation values of the given state, which gives us access to a great variety of non-classical states.

In the previous chapters we already discussed the coherent squeezed states as the most prominent candidate for squeezing but there are similar definitions for squeezed Fock state $|l\rangle_{s f}$ and squeezed thermal states $|n\rangle_{s t h}$ 47, 49.

$$
\begin{aligned}
|l\rangle_{s f} & =S(r, \phi)|l\rangle \\
|n\rangle_{s t h} & =S(r, \phi)|n\rangle .
\end{aligned}
$$

Both classes of states have already been investigated in some detail, with explicit expression for expectation values of all orders being known [38, [50. Hence, these squeezed Fock states can also be readily integrated into our variational approach.

Furthermore, it is also possible to employ highly entangled Schrödinger cat states given by

$$
|\psi\rangle=A\left(\left|\alpha_{1}\right\rangle+\Theta\left|\alpha_{2}\right\rangle\right),
$$

with $\left|\alpha_{1}\right\rangle$ and $\left|\alpha_{2}\right\rangle$ being two different coherent states. The expectation values for this state can be calculated via the explicit $\mathrm{P}$ distribution [51.

Fig. 5 shows the Wigner distribution of all three states. The Wigner distribution is more suitable for a visual representation because it does not have singularities for highly non-classical states that are found in the P distribution. The transformation

$$
W(\alpha)=\frac{2}{\pi} \int d^{2} \alpha^{\prime} e^{-2\left|\alpha-\alpha^{\prime}\right|^{2}} P\left(\alpha^{\prime}\right)
$$

connects both distributions.

We also want to make a clear distinction between the squeezed thermal (Fock) state and the convoluted distribution of a squeezed coherent state with a thermal (Fock) state. Especially in the case of the thermal state it is not straight forward to see from their Wigner functions that the two results are actually different. Therefore, it is instructive to look at the difference of their intensities, which is given by

$$
\left\langle a^{\dagger} a\right\rangle_{s t h}-\left\langle a^{\dagger} a\right\rangle_{s+t h}=2 n_{0} \sinh ^{2} r .
$$

The difference is even enhanced for higher-order expectation values, which can significantly change the result of the minimization in Eq. (14).

In case of the cat state the situation is reversed. Although the visual representation in 5 is cleary distinguishable from a simple coherent state, the difference enters only in higher orders, as the lowest order is given by $\langle a\rangle_{\text {cat }}=\alpha_{1}+\alpha_{2}=\tilde{\alpha}$. Only the scaling with higher order expectation values can reveal the true nature of this 


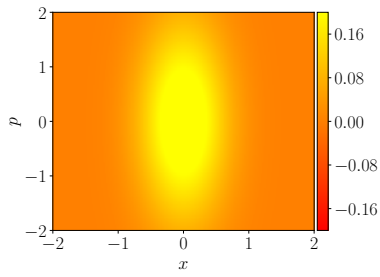

(a)

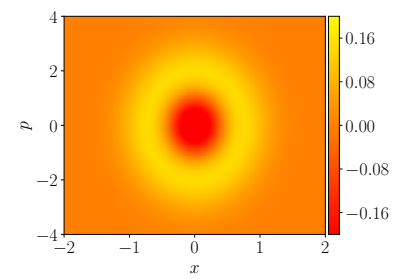

(b)

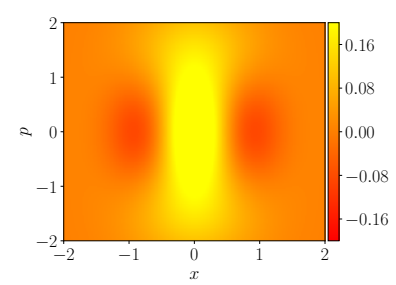

(c)

FIG. 5. Wigner function for different nonclassical states.(a) squeezed thermal state and (b) squeezed Fock state and (c) Schrödinger cat state.

state and shows the importance of incorporating as many orders as possible for the equations of motion.

Finally, we would like to mention two additional classes of states that could be included in the variational analysis. Both the single-variable Hermite polynomial states [26, 52, 54] and the photon-added (substracted) coherent states [24, 55] appear to be good candidate for a further expansion of the variational approach.

\section{SUMMARY}

In summary, we have extended the variational principle for open quantum systems through the usage of the $\mathrm{P}$ distribution of the density matrix. Despite its simplicity, we find that our method yields even quantitatively reasonable results for the driven-dissipative Jaynes-Cummings model. Furthermore, we have succesfully applied our approach to an effective model describe a many-body system of Rydberg atoms in a cavity, where we can identify strong squeezing of a dark state polariton mode. Our approach could be especially fruitful for applications where strong nonclassical correlations play an important role, such as gravitational wave detection using squeezed light [56 58 . or the preparation of nonclassical states of light in photonic condensates [59. Finally, we have presented several directions how the class of variational states could be extended further.

\section{ACKNOWLEDGMENTS}

We thank Jingtao Fan for fruitful discussions. This work was funded by the Volkswagen Foundation, by the Deutsche Forschungsgemeinschaft (DFG, German Research Foundation) within SFB 1227 (DQ-mat, project A04), SPP 1929 (GiRyd), and under Germany's Excellence Strategy - EXC-2123 QuantumFrontiers 390837967.
[1] J. Dalibard, Y. Castin, and K. Mølmer, Wave-function approach to dissipative processes in quantum optics, Phys. Rev. Lett. 68, 580 (1992).

[2] R. Dum, P. Zoller, and H. Ritsch, Monte Carlo simulation of the atomic master equation for spontaneous emission, Phys. Rev. A 45, 4879 (1992).

[3] J. Cui, J. I. Cirac, and M. C. Bañuls, Variational Matrix Product Operators for the Steady State of Dissipative Quantum Systems, Phys. Rev. Lett. 114, 220601 (2015).

[4] E. Mascarenhas, H. Flayac, and V. Savona, Matrixproduct-operator approach to the nonequilibrium steady state of driven-dissipative quantum arrays, Phys. Rev. A 92, 022116 (2015).

[5] J. J. Mendoza-Arenas, S. R. Clark, S. Felicetti, G. Romero, E. Solano, D. G. Angelakis, and D. Jaksch, Beyond mean-field bistability in driven-dissipative lattices: Bunching-antibunching transition and quantum simulation, Phys. Rev. A 93, 023821 (2016).

[6] A. Kshetrimayum, H. Weimer, and R. Orús, A simple tensor network algorithm for two-dimensional steady states, Nature Commun. 8, 1291 (2017).

[7] H. Weimer, A. Kshetrimayum, and R. Orús, Simulation methods for open quantum many-body systems, Rev. Mod. Phys. (to be published), arXiv:1907.07079.
[8] H. Weimer, Variational Principle for Steady States of Dissipative Quantum Many-Body Systems, Phys. Rev. Lett. 114, 040402 (2015).

[9] I. Carusotto and C. Ciuti, Quantum fluids of light, Rev. Mod. Phys. 85, 299 (2013).

[10] C. Aron, M. Kulkarni, and H. E. Türeci, PhotonMediated Interactions: A Scalable Tool to Create and Sustain Entangled States of $N$ Atoms, Phys. Rev. X 6, 011032 (2016).

[11] Y. O. Dudin and A. Kuzmich, Strongly Interacting Rydberg Excitations of a Cold Atomic Gas, Science 336, 887 (2012).

[12] T. Peyronel, O. Firstenberg, Q.-Y. Liang, S. Hofferberth, A. V. Gorshkov, T. Pohl, M. D. Lukin, and V. Vuletić, Quantum nonlinear optics with single photons enabled by strongly interacting atoms, Nature 488, 57 (2012).

[13] N. Jia, N. Schine, A. Georgakopoulos, A. Ryou, L. W. Clark, A. Sommer, and J. Simon, A strongly interacting polaritonic quantum dot, Nature Phys. 14, 550 (2018).

[14] A. V. Gorshkov, J. Otterbach, M. Fleischhauer, T. Pohl, and M. D. Lukin, Photon-Photon Interactions via Rydberg Blockade, Phys. Rev. Lett. 107, 133602 (2011).

[15] D. Tiarks, S. Schmidt-Eberle, T. Stolz, G. Rempe, and S. Dürr, A photon-photon quantum gate based on Rydberg interactions, Nature Physics 15, 124 (2019). 
[16] P. Bienias and H. P. Büchler, Two photon conditional phase gate based on Rydberg slow light polaritons, J. Phys. B 53, 054003 (2020).

[17] H.-P. Breuer and F. Petruccione, The Theory of Open Quantum Systems (Oxford University Press, Oxford, 2002).

[18] H. Weimer, Variational analysis of driven-dissipative Rydberg gases, Phys. Rev. A 91, 063401 (2015).

[19] V. R. Overbeck and H. Weimer, Time evolution of open quantum many-body systems, Phys. Rev. A 93, 012106 (2016).

[20] S. Ryl, J. Sperling, E. Agudelo, M. Mraz, S. Köhnke, B. Hage, and W. Vogel, Unified nonclassicality criteria, Phys. Rev. A 92, 011801 (2015).

[21] M. Bohmann, E. Agudelo, and J. Sperling, Probing nonclassicality with matrices of phase-space distributions, 2020.

[22] P. D. Drummond and C. W. Gardiner, Generalised Prepresentations in quantum optics, Journal of Physics A: Mathematical and General 13, 2353 (1980).

[23] M. S. Kim, W. Son, V. Bužek, and P. L. Knight, Entanglement by a beam splitter: Nonclassicality as a prerequisite for entanglement, Phys. Rev. A 65, 032323 (2002).

[24] A. Zavatta, V. Parigi, and M. Bellini, Experimental nonclassicality of single-photon-added thermal light states, Phys. Rev. A 75, 052106 (2007).

[25] A. I. Lvovsky and J. H. Shapiro, Nonclassical character of statistical mixtures of the single-photon and vacuum optical states, Phys. Rev. A 65, 033830 (2002).

[26] G. S. Agarwal and K. Tara, Nonclassical character of states exhibiting no squeezing or sub-Poissonian statistics, Phys. Rev. A 46, 485 (1992).

[27] R. J. Glauber, Coherent and Incoherent States of the Radiation Field, Phys. Rev. 131, 2766 (1963).

[28] E. C. G. Sudarshan, Equivalence of Semiclassical and Quantum Mechanical Descriptions of Statistical Light Beams, Phys. Rev. Lett. 10, 277 (1963).

[29] J. Johansson, P. Nation, and F. Nori, QuTiP: An opensource Python framework for the dynamics of open quantum systems, Comp. Phys. Comm. 183, 1760 (2012).

[30] J. Johansson, P. Nation, and F. Nori, QuTiP 2: A Python framework for the dynamics of open quantum systems, Comp. Phys. Comm. 184, 1234 (2013).

[31] E. T. Jaynes and F. W. Cummings, Comparison of quantum and semiclassical radiation theories with application to the beam maser, Proc. IEEE 51, 89 (1963).

[32] T. K. Mavrogordatos, Quantum phase transitions in the driven dissipative Jaynes-Cummings oscillator: From the dispersive regime to resonance, EPL (Europhysics Letters) 116, 54001 (2016).

[33] B. W. Shore and P. L. Knight, The Jaynes-Cummings Model, J. Mod. Opt. 40, 1195 (1993).

[34] H. J. Carmichael, Breakdown of Photon Blockade: A Dissipative Quantum Phase Transition in Zero Dimensions, Phys. Rev. X 5, 031028 (2015).

[35] G. Lindblad, On the generators of quantum dynamical semigroups, Commun. Math. Phys. 48, 119 (1976).

[36] H. Carmichael, Statistical Methods in Quantum Optics 1. Master Equations and Fokker-Planck Equations, 2002.

[37] R. Bonifacio, L. M. Narducci, and E. Montaldi, Conditions for Existence of a Diagonal Representation for Quantum Mechanical Operators, Phys. Rev. Lett. 16, 1125 (1966).
[38] P. Marian and T. A. Marian, Destruction of higher-order squeezing by thermal noise, Journal of Physics A: Mathematical and General 29, 6233 (1996).

[39] T. Kiesel, W. Vogel, B. Hage, J. DiGuglielmo, A. Samblowski, and R. Schnabel, Experimental test of nonclassicality criteria for phase-diffused squeezed states, Phys. Rev. A 79, 022122 (2009).

[40] C. Di Castro and R. Raimondi, Statistical Mechanics and Applications in Condensed Matter (Cambridge University Press, 2015).

[41] A. Grankin, E. Brion, E. Bimbard, R. Boddeda, I. Usmani, A. Ourjoumtsev, and P. Grangier, Quantum statistics of light transmitted through an intracavity Rydberg medium, New Journal of Physics 16, 043020 (2014).

[42] A. Grankin, E. Brion, E. Bimbard, R. Boddeda, I. Usmani, A. Ourjoumtsev, and P. Grangier, Quantumoptical nonlinearities induced by Rydberg-Rydberg interactions: A perturbative approach, Phys. Rev. A 92, 043841 (2015).

[43] T. Pistorius, J. Kazemi, and H. Weimer, Quantum manybody dynamics of driven-dissipative Rydberg polaritons, 2020.

[44] H. A. M. Leymann, A. Foerster, and J. Wiersig, Expectation value based cluster expansion, physica status solidi c 10, 1242 (2013).

[45] H. A. M. Leymann, A. Foerster, and J. Wiersig, Expectation value based equation-of-motion approach for open quantum systems: A general formalism, Phys. Rev. B 89, 085308 (2014).

[46] H. Schoeller, A New Transport Equation for SingleTime Green's Functions in an Arbitrary Quantum System. General Formalism, Annals of Physics 229, 273 (1994).

[47] P. Marian, Higher-order squeezing properties and correlation functions for squeezed number states, Phys. Rev. A 44, 3325 (1991).

[48] M. S. Kim, F. A. M. de Oliveira, and P. L. Knight, The Squeezing of Fock and Thermal Field States, in Coherence and Quantum Optics VI, edited by J. H. Eberly, L. Mandel, and E. Wolf, pages 601-605, Boston, MA, 1989, Springer US.

[49] M. S. Kim, F. A. M. de Oliveira, and P. L. Knight, Properties of squeezed number states and squeezed thermal states, Phys. Rev. A 40, 2494 (1989).

[50] P. Marian and T. A. Marian, Squeezed states with thermal noise. I. Photon-number statistics, Phys. Rev. A 47, 4474 (1993).

[51] R. A. Brewster and J. D. Franson, Generalized delta functions and their use in quantum optics, Journal of Mathematical Physics 59, 012102 (2018).

[52] M. Hillery, Amplitude-squared squeezing of the electromagnetic field, Phys. Rev. A 36, 3796 (1987).

[53] J. A. Bergou, M. Hillery, and D. Yu, Minimum uncertainty states for amplitude-squared squeezing: Hermite polynomial states, Phys. Rev. A 43, 515 (1991).

[54] T. R. Tan, J. P. Gaebler, Y. Lin, Y. Wan, R. Bowler, D. Leibfried, and D. J. Wineland, Multi-element logic gates for trapped-ion qubits, Nature 528, 380 (2015).

[55] S. M. Barnett, G. Ferenczi, C. R. Gilson, and F. C. Speirits, Statistics of photon-subtracted and photon-added states, Phys. Rev. A 98, 013809 (2018).

[56] Ligo Scientific Collaboration et al., A gravitational wave observatory operating beyond the quantum shot-noise limit, Nature Physics 7, 962 (2011). 
[57] Y. Zhao, N. Aritomi, E. Capocasa, M. Leonardi, M. Eisenmann, Y. Guo, E. Polini, A. Tomura, K. Arai, Y. Aso, Y.-C. Huang, R.-K. Lee, H. Lück, O. Miyakawa, P. Prat, A. Shoda, M. Tacca, R. Takahashi, H. Vahlbruch, M. Vardaro, C.-M. Wu, M. Barsuglia, and R. Flaminio, Frequency-Dependent Squeezed Vacuum Source for Broadband Quantum Noise Reduction in Advanced Gravitational-Wave Detectors, Phys. Rev. Lett. 124, 171101 (2020).

[58] L. McCuller, C. Whittle, D. Ganapathy, K. Komori, M. Tse, A. Fernandez-Galiana, L. Barsotti, P. Fritschel, M. MacInnis, F. Matichard, K. Mason, N. Mavalvala, R. Mittleman, H. Yu, M. E. Zucker, and M. Evans, Frequency-Dependent Squeezing for Advanced LIGO, Phys. Rev. Lett. 124, 171102 (2020).

[59] C. Kurtscheid, D. Dung, E. Busley, F. Vewinger, A. Rosch, and M. Weitz, Thermally condensing photons into a coherently split state of light, Science 366, 894 (2019). 


\section{Appendix A: Convoluted P distributions}

Tab. I shows examples of convolutions of two P distributions used in the main text.

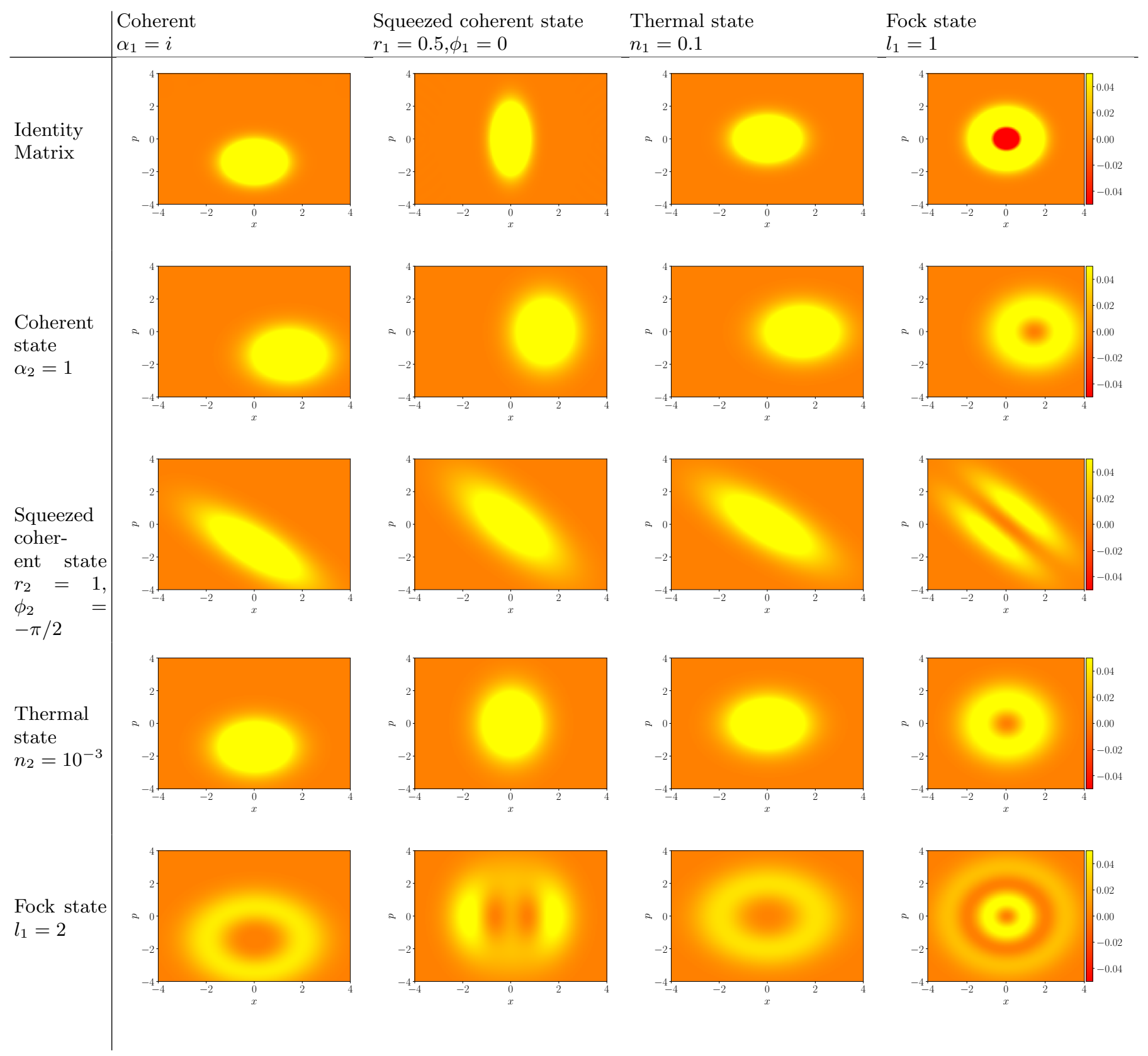

TABLE I. Convoluted Wigner functions of the coherent, squeezed coherent, thermal and Fock state with different parameters. 